\title{
Inventaire et vulnérabilité territoriale des basses terres du Saint-Laurent (Québec, Canada) face au projet d'exploitation des gaz de schiste
}

Inventory and territorial vulnerability of Saint-Laurent Lowlands (Quebec, Canada) to exploitation of shale gas project

Inventario y vulnerabilidad territorial de las tierras bajas del Saint-Laurent (Québec, Canadá) del proyecto de la explotación de gas de shale

\section{Christiane Gagnon et Majella-J Gauthier}

\section{(2) OpenEdition}

Journals

Édition électronique

URL : https://journals.openedition.org/ideas/1764

DOI : 10.4000/ideas.1764

ISSN : 1950-5701

Éditeur

Institut des Amériques

\section{Référence électronique}

Christiane Gagnon et Majella-J Gauthier, « Inventaire et vulnérabilité territoriale des basses terres du Saint-Laurent (Québec, Canada) face au projet d'exploitation des gaz de schiste », IdeAs [En ligne], 8 I 2016, mis en ligne le 21 décembre 2016, consulté le 18 octobre 2022. URL : http:// journals.openedition.org/ideas/1764; DOI : https://doi.org/10.4000/ideas.1764

Ce document a été généré automatiquement le 18 octobre 2022.

Creative Commons - Attribution - Pas d'Utilisation Commerciale - Pas de Modification 4.0 International - CC BY-NC-ND 4.0

https://creativecommons.org/licenses/by-nc-nd/4.0/ 


\title{
Inventaire et vulnérabilité territoriale des basses terres du Saint-Laurent (Québec, Canada) face au projet d'exploitation des gaz de schiste
}

\author{
Inventory and territorial vulnerability of Saint-Laurent Lowlands (Quebec, \\ Canada) to exploitation of shale gas project \\ Inventario y vulnerabilidad territorial de las tierras bajas del Saint-Laurent \\ (Québec, Canadá) del proyecto de la explotación de gas de shale
}

Christiane Gagnon et Majella-J Gauthier

Nos remerciements vont aux personnes qui ont travaillé à la réalisation de l'étude, particulièrement Erik Langevin, Carl Brisson ainsi que Daniel Beaulieu-Gagnon, Raphaël Gadbois-Langevin, Mélanie Lambert, Carl Simard et Jonathan Skeene-Parent. Sans oublier les collaborateurs ponctuels tels les fonctionnaires gouvernementaux et le personnel des Municipalités Régionales de Comté.

\section{Mise en contexte de la recherche}

L'exploitation des gaz et du pétrole de schiste aux États-Unis (ÉU) et au Canada, selon la nouvelle technologie de fracturation hydraulique, est venue changer la donne du portrait énergétique national. Il est prévu que les ÉU atteignent une indépendance énergétique face au pétrole. Le Québec, importateur de pétrole et de gaz (30 Mtep, chiffres de 2009) ${ }^{1}$, n'échappe pas à l'attrait de l'exploitation de ses ressources provenant du pétrole et des gaz de schiste. La découverte du gisement d'Utica, qui recouvre une grande partie habitée du sud-ouest de la province de Québec (figure 1), a 
entraîné, à l'abri des regards, la vente des droits d'exploration aux multinationales minières.

Figure 1: Limites de la zone à potentiel gazier

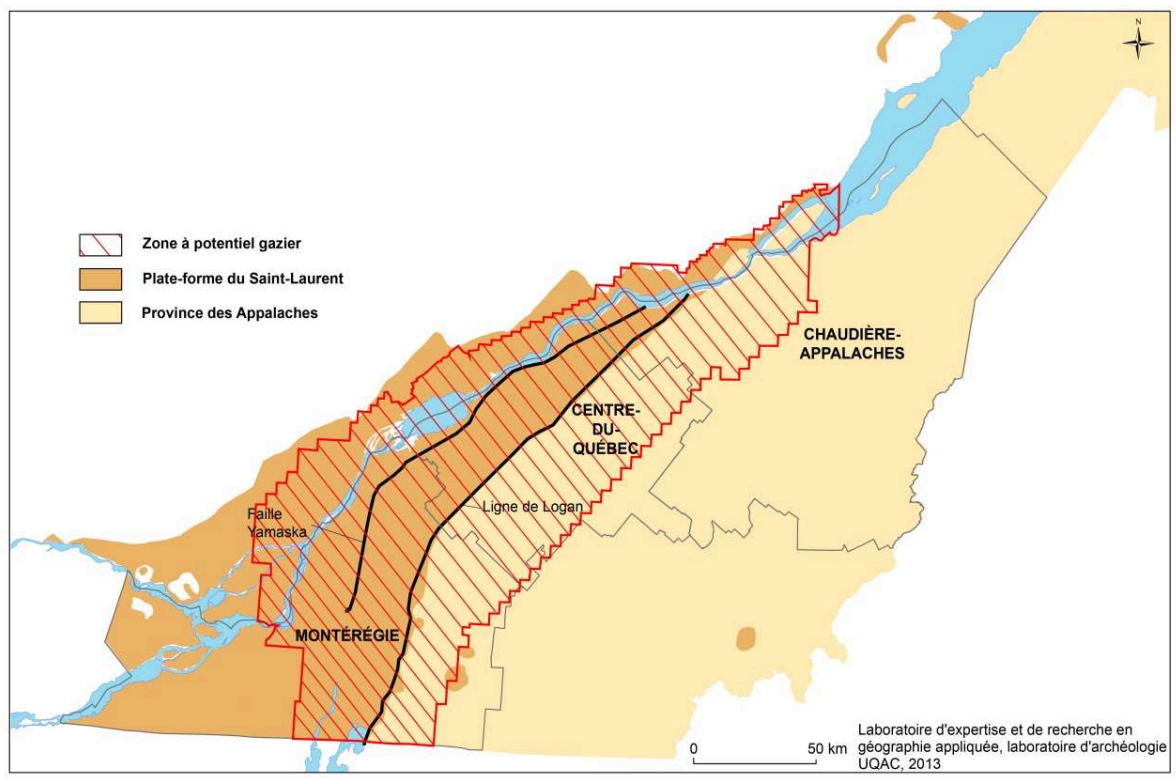

SOURCE : GAgNON C., $2013: 42$.

2 Toutefois, les premières opérations d'exploration en milieu urbain et rural ont soulevé l'ire de la société civile et des municipalités. Devant l'ampleur de la contestation des maires et des groupes de citoyens, le gouvernement du Québec, via le Bureau des audiences publiques sur l'environnement (BAPE), lance une première consultation publique (2011) afin de définir seulement des règles d'encadrement pour l'industrie. Dans son rapport, Le développement durable de l'industrie des gaz de schiste au Québec, le BAPE révèle un certain nombre d'impacts possibles sur la qualité de vie des citoyens et la qualité de l'environnement (BAPE 2011). Face au manque de connaissances sur les impacts de cette nouvelle industrie, il propose la réalisation d'une évaluation environnementale stratégique (ÉES) par un comité multipartite. Ce comité, composé de tous les acteurs sociaux, privés et d'universitaires,a élaboré un Plan²afin de combler les connaissances manquantes. Ainsi, 73 études ont été commandées, majoritairement aux experts universitaires. Une synthèse a été publiée en 2014 afin de vulgariser et diffuser les connaissances acquises. Une de ces études prévoyaitl'inventaire territorial et l'évaluation $\mathrm{du}$ potentiel archéologique des régions concernées (figure 2), précédemment à toute exploitation industrielle. 
Figure 2: Limites du territoire et identification des $30 \mathrm{MRC}$ à l'étude

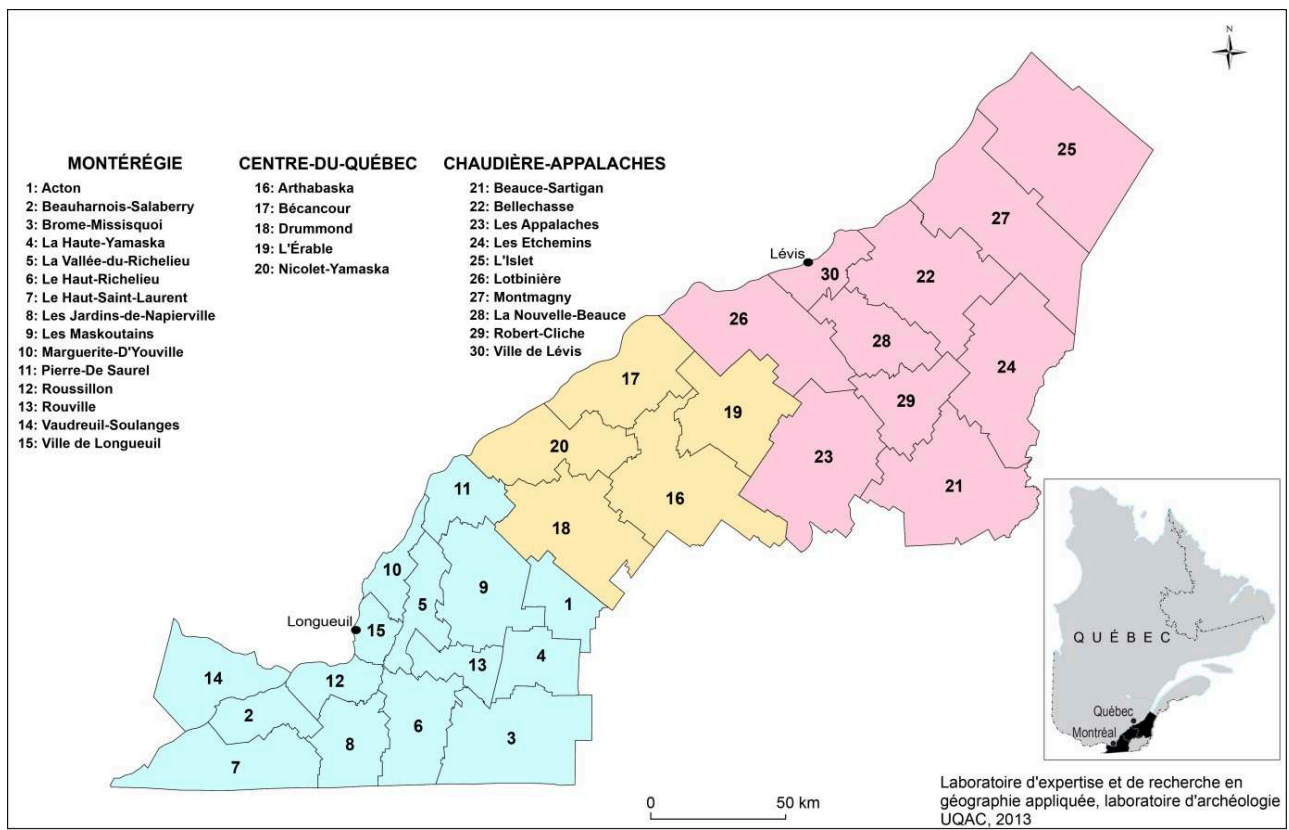

SOURCE : GAgNON C., $2013: 13$.

Dans le présent article, nous discuterons surtout de la méthodologie employée pour réaliser cet inventaire, des résultats originaux obtenus et de la réflexion qui s'ensuit, dans une optique de planification et d'aménagement viable du territoire (Gagnon, 2013). Le contexte sociopolitique d'exploitation du pétrole et des gaz de schiste étant relativement récent et faisant l'objet de nombreuses controverses, tant dans le monde qu'au Québec (instauration d'un moratoire en 2013, audiences publiques en 2014, projet de Loi en 2016), l'ÉES québécoise a été l'une des premières à documenter les impacts de cette nouvelle industrie. Une des premières étapes, nécessaire aussi à d'autres études, est de connaître l'état initial des usages territoriaux actuels, soit avant toute modification, d'où la pertinence de l'inventaire territorial et la détermination des aires de sensibilité.

\section{Problématique et méthodologie}

L'étude qui a été réalisée répond à une des questions de base de toute évaluation environnementale, à savoir quel est l'état initial de l'aménagement du territoire visé par ce projet d'exploitation gazière et quel serait l'impact de cette industrie sur les usages existants?

Deux objectifs spécifiques ont été poursuivis par l'étude sur l'inventaire territorial, soit identifier : 1) des aires et des secteurs de sensibilité à l'échelle des 30 Municipalités Régionales de Comtés (MRC), comprises dans 3 des 17 régions administratives du Québec, et 2) un niveau de vulnérabilité territoriale à l'échelle interrégionale.D'entrée de jeu, mentionnons que l'approche méthodologique est descriptive, empirique et systémique, compte tenu de l'objectif descriptif de l'inventaire territorial. Pour l'ensemble du territoire à l'étude, soit $34672 \mathrm{~km}^{2}$, onze composantes ont été préalablement déterminées. Ces composantes recoupent l'ensemble des usages territoriaux en cours (voir rectangles de couleur dans lafigure 3). C'est à l'aide d'un 
système d'information géographique (SIG, ArcGis) que le regroupement, l'intégration et l'analyse d'environ 1,5 million de données ont été faits.

6 L'unité cartographique de base choisie est la MRC. C'est une entité administrative responsable de l'aménagement du territoire : elle regroupe en général un centre urbain de taille moyenne ${ }^{3}$ et des municipalités rurales ou résidentielles. La superficie moyenne d'une MRC au Québec est de $16000 \mathrm{~km}^{2}$. Pour chaque MRC, les données colligées ont étédéclinées en onze composantes territoriales: 1) l'espace urbanisé, 2) l'espace agricole, 3) le potentiel agricole, 4) les milieux humides, 5) les aires protégées, 6) l'espace forestier, 7) les érablières, 8) l'hydrographie, 9) les sites archéologiques et les monuments historiques, 10) le potentiel archéologique, 11) les territoires d'intérêt régionaux: historique, culturel, esthétique et écologique (figure 3).

Figure 3: Cadre méthodologique et opérationnel

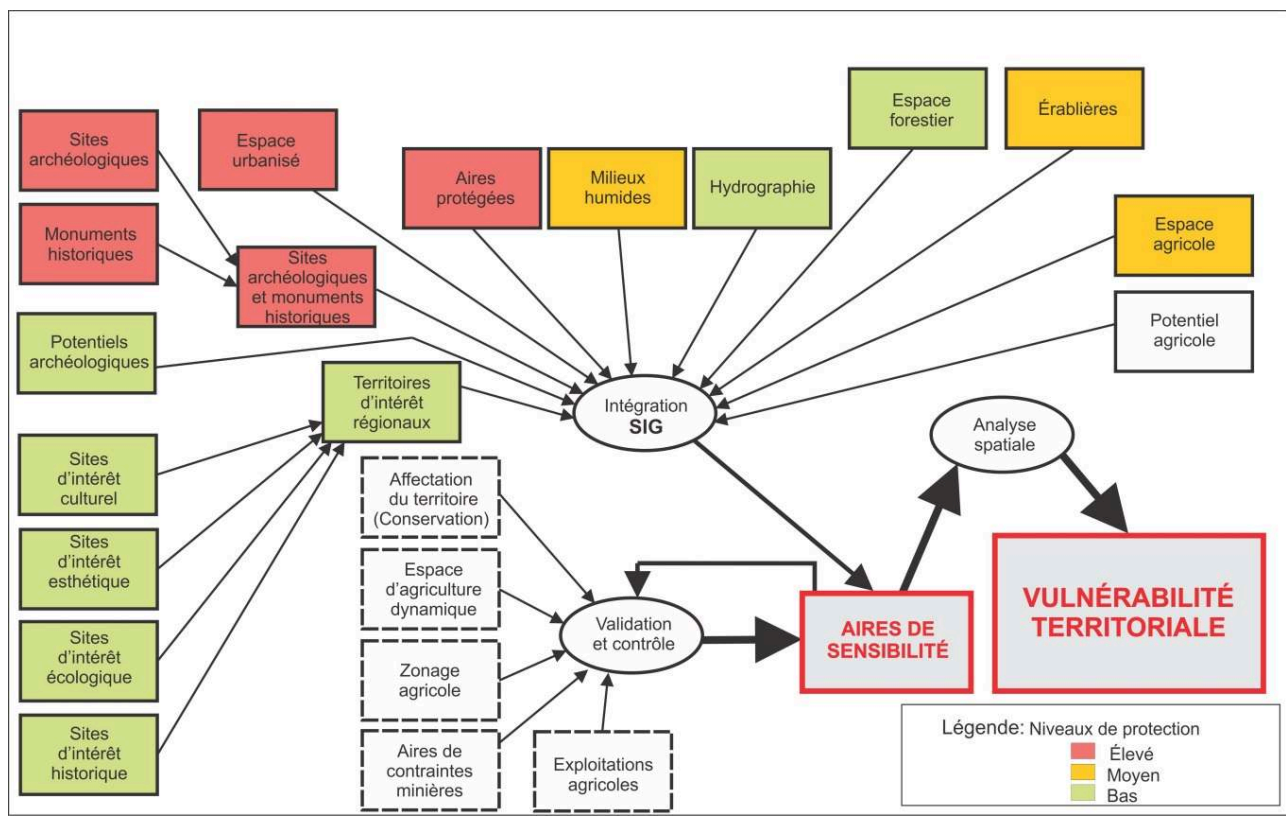

SOURCE : GAgNON, C., $2013: 11$.

7 Les données géo-référencées, relatives à ces composantes, proviennent principalement de la base de données du ministère des Affaires municipales, des Régions et de l'Occupation du Territoire (MAMROT), et de celles produites par une douzaine de ministères et d'organisations privées et publiques. Toutefois, les données ne sont pas toutes de la même année, car la révision décennale des schémas d'aménagement ou la collecte des données secondaires varient selon les situations ou les organisations. L'architecture du nouveau SIG ainsi constitué permettait l'intégration et l'analyse spatiale des nombreuses données, mais aussi la production de nouvelles informations et de cartes. Les cartes originales de base ont été converties en modèle matriciel, composé de cellules de 500 mètres par 500 mètres (ArcGis), ce qui permet une précision (localisation et finesse) dans l'inventaire et une analyse des aires de sensibilité.

8 Celles-ci ont été déterminées sur la base de la compilation des usages et des composantes territoriales présentes dans une même cellule, six usages étant le maximum de composantes retrouvé pour une même cellule. La notion de sensibilité territoriale est définie ici comme une portion de territoire où il $\mathrm{y}$ a des usages 
territoriaux concomitants, valorisés par des acteurs, mais où tout ajout ou toute perturbation dans lesdits usages entraîne une modification, une pression supplémentaire sur les composantes du territoire, avec des conséquences sur l'aire donnée ou les aires voisines. Certaines études ont été utiles du point de vue d'une telle définition de la notion d'aires de sensibilité, notamment celle sur l'éolien (Bouchard G., Villeneuve C. et Pelletier L., 2007).

9 Par la suite, une catégorisation des aires a été faite pour qualifier leur niveau de sensibilité.Cette catégorisation s'est basée sur la présence et la combinaison d'un nombre différencié de composantes (de 1 à 6) sur un même espace. Par la suite, elles ont été divisées en trois catégories: 1 ) sensible (1-2 composantes), 2) très sensible (3-4 composantes), 3) extrêmement sensible (5-6 composantes). La catégorie de l'aire extrêmement sensible comprend aussi le statut légal de protection intégrale de certaines composantes, telles que les réserves écologiques et les parcs nationaux. Dans ce sens, une certaine pondération a été faite par les chercheurs entre les composantes et leur statut légal.

10 De même, une opération de sommation des usages a été réalisée pour chaque cellule ainsi que des cartes pour chacune des 30 MRC à l'étude. Cependant, en cours de recherche, la seule détermination des aires de sensibilité est apparue comme incomplète, d'où les questions suivantes qui ont orienté la réflexion: comment ces aires étaient-elles reliées entre elles? Comment interpréter leur présence, eu égard au changement planifié et à la gestion des risques? L'impact de la somme de ces aires étaitil cumulatif? Y avait-il à l'échelle régionale une plus ou moins grande vulnérabilité selon les différenciations territoriales?

11 La problématique de recherche s'est alors enrichie du concept de vulnérabilité territoriale, notion plus complexe et interprétative (voir figure 3). Il correspond ici à un espace vécu englobant un cumul de composantes et d'enjeux territoriaux, de divers niveaux de sensibilité, cumul soumis à l'exposition d'une perturbation humaine. Nous appuyant notamment sur les recherches de Robert D'Ercole et Pascale Metzger (2009), nous postulons "qu'il existe, au sein de tout territoire, des éléments localisables susceptibles d'engendrer et de diffuser leur vulnérabilité à l'ensemble d'un territoire, provoquant des effets qui peuvent perturber, compromettre, voire interrompre son fonctionnement et son développement ». Il faut cependant appréhender la notion de vulnérabilité avec souplesse (Conseil général de Haute-Savoie, 2007); les niveaux d'aires de sensibilité et la vulnérabilité expriment davantage une difficulté, voire une impossibilité à harmoniser, à concilier les usages territoriaux.

Cette notion a permis de mobiliser l'ensemble des composantes à l'étude et l'impact sur ces dernières lorsque celles-ci sont soumises à un changement ou à une perturbation externe, ici l'exploration et l'exploitation industrielles des gaz de schiste (Magnan A., 2009). De même, en fouillant la littérature sur la vulnérabilité territoriale, à travers les corpus de la gestion des risques et de l'évaluation des incidences sociales des changements majeurs, la notion du cumul des enjeux territoriaux permettait d'ajouter à la compilation quantitative et cartographique des composantes territoriales, une dimension qualitative. Pour évaluer ces enjeux par MRC, et compte tenu du temps court imparti pour la réalisation de cette étude (6 mois), nous sommes partis de l'analyse de contenu de leur schéma d'aménagement pour déduire les enjeux sociaux, environnementaux et économiques (forestier et agricole) spécifiques, relatifs à la cohabitation ou des usages ou au développement durable. Les enjeux ont été considérés 
comme des construits d'acteurs portant ou non des intérêts communs ou contradictoires (AEA Technology, 2012; Grieg G., 2011; NYSDEC, 2009; Canada, 1999).Ces enjeux, selon les acteurs, pouvaient donc être soit des contraintes soit des potentiels. Par exemple, l'usage agricole et protégé par la Loi d'une terre aux aptitudes agricolespeut représenter une contrainte pour un promoteur, mais un potentiel pour l'agriculteur. Ces enjeux ont été croisés avec les aires de sensibilité et leur sommation (voir figure 5).

13 L'opération de zonage a commencé par la délimitation de grandes aires homogènes d'utilisation du sol, définies par la carte de l'utilisation majeure du sol pour l'ensemble du territoire. De là étaient dégagés des ensembles comme les paysages à dominance agricole, forestière, urbanisée et d'utilisation mixte. Puis, sont intervenues d'autres dimensions:

- la superposition de l'aire géologique à potentiel de gaz de schiste permettant un redécoupage de certaines zones de manière à ce que chacune d'elles soit complètement comprise ou non dans cette aire;

- la répartition des aires de sensibilité du territoire;

- la présence et la dominance des enjeux de développement durable. Le résultat final prend la forme d'une représentation cartographique, voire un modèle, rassemblant chacune des quatre variables.

\section{Résultats de l'inventaire territorial}

Les principaux résultats concernent la détermination des aires de sensibilité pour chacune des 30 MRC à l'étude et la zone de vulnérabilité à l'échelle interrégionale. Dans un premier temps, quels constats pouvons-nous faire?

Pour l'ensemble des trois régions québécoises concernées par le projet d'exploitation des gaz de schiste, la superficie occupée par les aires de sensibilité de catégorie sensible couvre $83 \%$, soit $29336 \mathrm{~km}^{2}$ alors que la catégorie très sensible représente $9 \% \mathrm{du}$ territoire à l'étude, soit $3242 \mathrm{~km}^{2}$ (figure 4). La catégorie extrêmement sensible occupe $7 \%$ du territoire, soit $2404 \mathrm{~km}^{2}$ et se localise particulièrement le long du corridor de la rive sud du fleuve Saint-Laurent.

Du point de vue de la répartition spatiale des aires de sensibilité, notons qu'à l'intérieur des terres, la sensibilité varie beaucoup, tout en se caractérisant par une prédominance des aires de catégorie sensible. Cependant, plusieurs espaces dont la sensibilité est extrême sont facilement repérables dans le centre et le nord de la région à l'étude, même si les espaces forestiers dominent. Au sud, il est possible d'observer un réseau linéaire d'aires très sensibles. 
Figure 4: Aires de sensibilité dans le territoire à l'étude

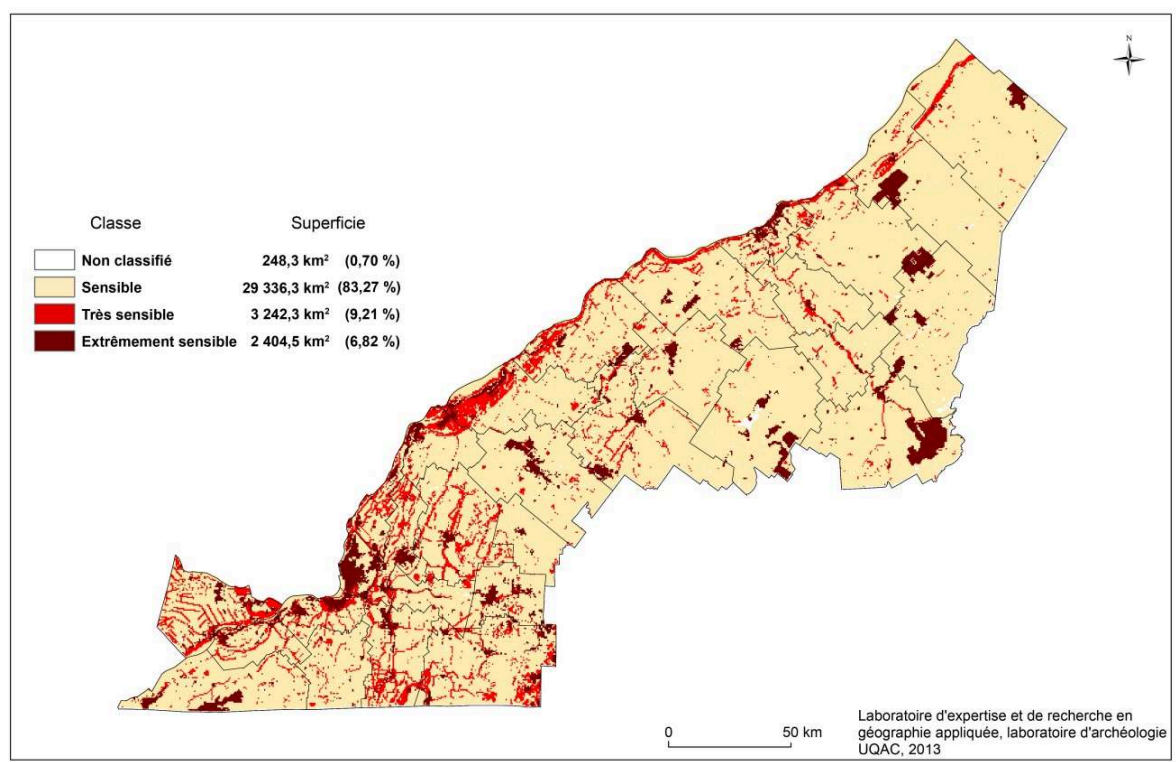

SOURCE : GAgNON C., 2013 : 12. évaluation environnementale par projet, ne peuvent être dissociées d'une analyse subséquente et cumulative, des incidences d'une exploitation industrielle ou d'un changement planifié. Par exemple, dans de conditions éco-géographiques communes, tel un bassin versant, il ne faut pas oublier que ces aires sont inter reliées, agissant comme des vases communicants, selon des principes d'interdépendance et de connectivité entre les aires voisines ou contiguës. Une aire sensible qui se retrouve encerclée par des aires extrêmement sensibles, telle l'île Perrot à l'est de la MRC de Vaudreuil-Soulanges, mériterait une attention particulière dans l'évaluation des impacts, lors d'une perturbation planifiée ou non. Toutefois, n'en rester qu'à l'identification des aires de sensibilité n'aurait pas pu mener à une synthèse et à une interprétation plus globale du territoire possiblement soumis à des nouveaux changements externes d'envergure, soit ceux de l'industrie pétrolière et gazière.

Dans un deuxième temps, examinons les résultats concernant la vulnérabilité territoriale. La figure 5 permet d'identifier visuellement le découpage de 18 zones de vulnérabilité. Tout d'abord, nous avons distingué celles potentiellement impactées des autres. Ainsi, huit zones seulement sont exposées au potentiel d'exploitation des schistes et couvrent $16326 \mathrm{~km}^{2}$, soit $46 \%$ du territoire. Selon les données du ministère de l'Énergie et des Ressources naturelles sur la nature du gisement d'Utica, dix autres zones sont situées à l'extérieur de la zone potentielle d'exploitation soit une superficie de $18951 \mathrm{~km}^{2}$ (54 \% du territoire). Le niveau de sensibilité est en général plus élevé dans les zones exposées potentiellement que dans les zones non exposées. Toutefois, il faut souligner que les zones non exposées, mais limitrophes aux zones exposées, en raison de leur proximité, voire connectivité, pourraient être sujettes à la vulnérabilité de voisinage.

19 À l'analyse, celles qui ressortent comme les plus vulnérables longent le fleuve SaintLaurent; elles comportent davantage d'enjeux sociaux, environnementaux, de 
cohabitation des usages et sont sujettes à des niveaux élevés de sensibilité. Elles couvrent grosso modo un espace partant du lac Champlain et allant jusqu'à l'île d'Orléans près de Québec. L'arrière-pays est plus agricole, et ne comporte le plus souvent que cette composante. Au total, dix MRC sont comprises dans le bloc des zones possiblement perturbées tandis que onze MRC le sont partiellement.

Figure 5: Vulnérabilité territoriale interrégionale

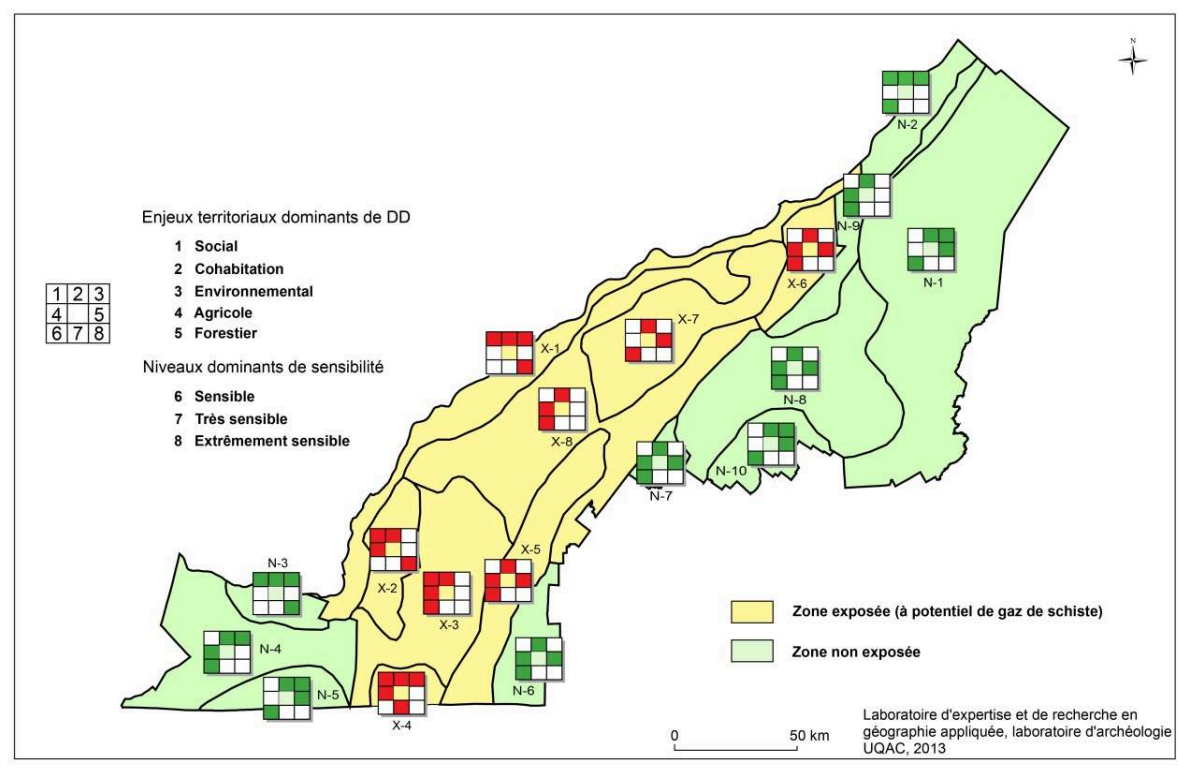

SOURCE :GAgNON C., $2013: 14$

Les zones non exposées, en théorie, se divisent en trois blocs géographiques. Celui à l'est est le plus important en surface ; là, les enjeux forestiers et agricoles dominent et le niveau de sensibilité est relativement bas. Le bloc à l'ouest diffère un peu des autres, dans la mesure où la zone à la confluence du fleuve Saint-Laurent et la rivière des Outaouais présente les mêmes caractéristiques que celles longeant le fleuve en aval. Le troisième bloc longe la frontière des États-Unis centrée autour d'East-Hereford.

Chacune des dix-huit zones, déterminées selon les données obtenues sur les usages et le portrait social et économique pour chaque MRC, comprend un domino représentant les cinq niveaux de sensibilité et les cinq types d'enjeux territoriaux, selon leur dominance, recensés suite à l'analyse de contenu de tous les schémas d'aménagement de la zone à l'étude. Leur définition inductive, c'est-à-dire résultant de la rechercheprovenant des données empiriques, est la suivante:

1) l'enjeu social concerne davantage l'environnement, la santé, la qualité de vie, la perte du patrimoine historique et culturel et la perte de paysages bâtis et naturels,

2) l'enjeu de cohabitation concerne des usages liés au territoire agricole et àson homogénéité, aux sites patrimoniaux et paysagers, aux milieux naturels et à l'apport économique de l'agriculture,

3) l'enjeu environnemental fait état de la perte des milieux naturels, de la biodiversité et des milieux humides, 
25 4) l'enjeu agricole touche la perte d'espaces et des activités et dans une moindre mesure la pérennité de l'agriculture, deux volets. D'une part, il y a eu la création d'une base de données géo-référencées unique, intégrant l'ensemble des composantes territoriales de trois régions québécoises et, d'autre part, le développement d'une méthodologie pour la détermination des aires de sensibilité et de vulnérabilité territoriale. En effet, celle-ci pourrait servir lors des évaluations environnementales subséquentes ainsi que lors de l'exercice légal par les MRC de la détermination des zones compatibles pour les activités humaines et résidentielles, lors du zonage d'usages industriels ou encore dans la gestion des risques et des plans d'urgence.

Malgré le potentiel de développement et la pertinence de cet inventaire territorial, des limites demeurent. Tout d'abord, l'étude n'est qu'une partie d'un tout; il y manque la vision globale intégrant les autres dimensions de l'ÉES et du Plan gouvernemental d'acquisition des connaissances ${ }^{5}$. Puis, du point de vue temporel, les données et l'information n'étaient pas uniformes quant à l'année de référence, soit 2011. Également, l'emploi du format matriciel a imposé un découpage du territoire en cellules. Or, c'est un découpage purement géométrique qui ne tient compte ni des limites naturelles et écologiques ni du terrain et de sa topographie. De même, la diminution de la précision cartographique a parfois amené une réduction de la signification de l'information. Par exemple, une cellule qui contenait initialement trois sites archéologiques est devenue un espace où il n'était possible de mesurer que leur présence ou absence.Enfin, l'apparition de quelques doublons dans les données n'a pu être totalement évitée ; malgré tout, pour l'ensemble du territoire, il nous sembleque cela ne modifierait pas de façon significative les résultats finaux. Toutefois, une 
validation terrain avec tous les services d'aménagement des MRC permettrait une plus grande précision.

L'inventaire territorial, bien que recouvrant l'ensemble des usages (99\% du territoire), a été fait à partir des composantes ou variables introduites dans l'architecture de base du SIG. Les données hydrogéologiques, la localisation des sites d'enfouissement des déchets dangereux, les caractéristiques paysagères, les contraintes naturelles (glissement de terrain, zone inondable, zone d'érosion, tremblement de terre), les autres utilisations $\mathrm{du}$ sol telles qu'industrielle, touristique, commerciale, institutionnellen'ont pas été incluses dans l'analyse, malgré leur pertinence.

Le potentiel de développement de l'outil est fort intéressant. Par exemple, il serait possible de croiser la base de données avec les données à caractère social, environnemental et économique, données produites par les autres équipes participant à l'ÉES. Ainsi, les aires et les secteurs de sensibilité et l'analyse spatiale de la vulnérabilité territoriale seraient confrontés, précisés et nuancés. Enfin, l'analyse de contenu des enjeux territoriaux, à partir d'un seul type de documents, soit les schémas d'aménagement, mériterait d'être validée auprès de la société civile et de ses acteurs.

Du point de vue cartographique, la contribution est double: 1) l'intégration des données afférentes aux composantes territoriales dans un SIG, dédié à un vaste territoire, 2) la définition d'aires de sensibilité et, enfin, l'identification d'une vulnérabilité territoriale dans un contexte d'exploitation industrielle.

En bref, en combinant l'analyse des aires de sensibilité à celle de la vulnérabilité territoriale interrégionale, nous avons tenté d'éviter le piège de la simple description, normalement rattachée à un inventaire de ce type, pour donner une cohérence, un sens à l'ensemble des données, compte tenu des enjeux soulevés par le développement gazier et pétrolier et leur confrontation aux objectifs de développement durable, et ce pour les populations locales et humaines, présentes et futures.

\section{BIBLIOGRAPHIE}

AEA Technology, Support to the identification of potential risks for the environment and human health arising from hydrocarbons operations involving hydraulic fracturing in Europe, Report for European Commission DG Environment, 2012, 292 p., http://ec.europa.eu/environment/ integration/energy/pdf/fracking\%20study.pdf.

BAPE, Développement durable de l'industrie de gaz de schiste au Québec, rapport d'enquête et d'audience publique (rapport n²73), gouvernement du Québec, 2011, 336 p., http:// www.bape.gouv.qc.ca/sections/rapports/publications/bape273.pdf.

Bouchard, Guy, Claire Villeneuve et Louise Pelletier, Analyse territoriale, volet éolien, ChaudièreAppalaches, Direction de l'énergie, des mines et du territoire public de la Capitale-Nationale et de la Chaudière - Appalaches, Ministère des Ressources naturelles et de la Faune du Québec, 2007, http://mern.gouv.qc.ca/publications/territoire/planification/analyse_territoriale_chaudiereappalaches.pdf. 
Groupe de travail sur l'évaluation des effets cumulatifs et AXYS Environmental Consulting Ltd, Guide du praticien sur l'évaluation des effets cumulatifs, Agence canadienne d'évaluation environnementale, Canada, 1999, http://www.ceaa.gc.ca/default.asp?lang=Fr\&n=43952694-1.

Conseil général de Haute-Savoie, Schéma départemental des espaces naturels sensibles de Haute-Savoie 2008-2014, Annecy, 2007, 56 p.

D’Ercole, Robert et Pascale Metzger, « La vulnérabilité territoriale: une nouvelle approche des risques en milieu urbain ». Cybergeo : European Journal of Geography [en ligne], Dossiers, Vulnérabilités urbaines au sud, article 447, 2009, mis en ligne le 31 mars, consulté le 27 décembre 2012. URL : http://cybergeo.revues.org/22022.

Gagnon, Christiane et collaborateurs, Inventaire territorial des régions québécoises ayant un potentiel d'exploitation des gaz de schiste, Université du Québec à Chicoutimi, Laboratoire d'expertise et de recherche en géographie appliquée (LERGA), rapport réalisé pour le ministère du Développement durable, de l'Environnement, de la Faune et des Parcs du Québec, 2013, 555 p. http://www.bape.gouv.qc.ca/sections/mandats/gaz_de_schiste-enjeux/documents/PR3.7.10.pdf.

Grieg, George, Marcellus shale advisory commission, Local impact and emergency response, work group recommendation format, Pennsylvania Department of Environmental Protection, 2011, 76 p., http://files.dep.state.pa.us/PublicParticipation/MarcellusShaleAdvisoryCommission/ MarcellusShaleAdvisoryPortalFiles/Local_Impacts_Emergency_Response.pdf.

Magnan, Alexandre, «La vulnérabilité des territoires littoraux au changement climatique: Mise au point conceptuelle et facteurs d'influence ", Iddri, Institut du développement durable et des relations internationales, $\mathrm{N}^{\circ}$ 01/2009,Changement climatique, 2009, 30 p. http://www.iddri.org/ Publications/Collections/Analyses/An_0901_A.Magnan_Vulnerabilite.pdf.

NYSDEC, Well Permit Issuance for Horizontal Drilling And High-Volume Hydraulic Fracturing to Develop the Marcellus Shale and Other Low-Permeability Gas Reservoirs, Bureau of oil \& gas regulation NYSDEC division of mineral resources, Supplemental Generic Environmental Impact Statement On The Oil, Gas and Solution Mining Regulatory Program, New York State, Department of Environmental Conservation, Draft, 2009, 804 p.

Québec, Plan de réalisation de l'évaluation environnementale stratégique sur les gaz de schistes, Comité de l'évaluation environnementale stratégique sur les gaz de schistes, version finale, avril 2012, 84 p. http://www.mddelcc.gouv.qc.ca/evaluations/Gaz-de-schiste/plan-realisation.pdf.

Québec, Rapport synthèse: évaluation environnementale stratégique sur le gaz de schiste, Comité de l'évaluation environnementale stratégique sur le gaz de schiste, 2014, http://

www.mddelcc.gouv.qc.ca/evaluations/Gaz-de-schiste/rapport-synthese.pdf.

\section{NOTES}

1. Commission sur les enjeux énergétiques du Québec, 2013.

2. Pour plus d'information sur le Plan de réalisation de l'ÉES sur les gaz de schistes; voir le site du BAPE.

3. Parmi ce nombre, il y a les Villes de Longueuil et de Lévis qui sont aussi des MRC.

4. Mentionnons que ces composantes étaient en majeure partie déterminées dans le mandat. Pour plus de renseignements sur le cadre conceptuel et méthodologique de l'inventaire territorial, voir l'étude au site suivant: http://www.bape.gouv.qc.ca/sections/mandats/ gaz_de_schiste-enjeux/documents/PR3.7.10.pdf 
5. Il est à noter que le rapport synthèse produit par le Comité de l'évaluation environnementale stratégique a été déposé en janvier 2014 (Québec, 2014); il reprend en grande partie les conclusions de l'étude réalisée par les auteurs de l'Université du Québec à Chicoutimi.

\section{RÉSUMÉS}

Dans le cadre du Plan de réalisation de l'évaluation environnementale stratégique sur les gaz de schiste (Gouvernement du Québec, 2011),73 études scientifiques ont été réalisées entre 2013 et 2014, tant sur les dimensions sociale, économique qu'environnementale. L'une d'entre elles, menées par les auteurs de cet article, visait à procéder à un inventaire du territoire couvrant l'ensemble des usages territoriaux des trois régions administratives québécoises, concernées par une éventuelle exploitation des gaz de schiste. Elle répond à une des questions de base de toute évaluation environnementale, à savoir quel est l'état initial de l'aménagement du territoire visé par ce projet d'exploitation gazière et quel en serait l'impact de cette industrie sur les usages existants ? Le présent travail résume le volumineux rapport de recherche répondant à un mandat du gouvernement du Québec. L'article est centré sur la méthodologie employée pour réaliser cet inventaire et sur les résultats obtenus au niveau de l'identification des aires de sensibilité, à l'échelle microrégionale, et de la vulnérabilité territoriale régionale. En conclusion, un certain nombre d'applications futures pour l'aménagement et la gestion du territoire sont dégagées.

In the framework of the Application plan of the Strategic Environmental Assessment on Shale Gas (Government of Quebec, 2011), 73 scientific studies were conducted between 2013 and 2014 to investigate social, economic and environmental issues. One of them, which was conducted by the authors of this article, was meant to produce an inventory of the territory covering all land uses in three Quebec administrative regions, concerned by possible shale gas exploitation. It answers two basic questions of all environmental assessments, namely: what is the initial state of the land used involved in this gas development project and what would be the impact of this industry on existing uses? This article summarizes the voluminous research report responding to a mandate from the Quebec government. It focuses on the methodology used and on the main outcomes of the study regarding the identification of sensitive areas, at a micro-regional level, and the regional territorial vulnerability. In conclusion, a number of applications for future planning and management of territory are identified.

En el marco de la implementación de la evaluación estratégica ambiental sobre el gas de esquisto (Gobierno de Quebec, 2011), 73 estudios científicos fueron realizados entre 2013 y 2014, tanto en el ámbito social, económico y ambiental. Uno de estos estudios tenía por objetivo hacer un inventario del territorio que cubre la totalidad de la tierra utilizada en tres regiones administrativas de Quebec involucradas por una eventual explotación de gas de esquisto. Este estudio responde a la pregunta básica de cualquier evaluación ambiental, a saber, cuál es el estado inicial del territorio que cubre este proyecto de explotación de gas y cuál sería el impacto de esta industria sobre los usos de la tierra ya existentes? El presente artículo resume un voluminoso informe de investigación respondiendo a un mandato del gobierno de Quebec. Este se centra en el resultado principal del estudio y la metodología, sea la identificación de las áreas sensibles a nivel micro-regional y la vulnerabilidad territorial regional. En conclusión, una serie de aplicaciones futuras para la planificación y la gestión del territorio fueron generadas. 
INDEX

Keywords : inventory, uses of territory, issues, shale gas, zone of sensitivity, vulnerability, Quebec

Mots-clés : inventaire, territoire, enjeux, gaz de schiste, zone de sensibilité, vulnérabilité, Québec

Palabras claves : inventario, territorio, desafios, gas de shale, zona de sensibilidad, vulnerabilidad, Quebec

\section{AUTEURS}

\section{CHRISTIANE GAGNON}

Christiane Gagnon est professeure à l'Université du Québec à Chicoutimi (UQAC). Elle est détentrice d'un Ph.D. en aménagement et d'un postdoctorat sur la question de la méthodologie de l'évaluation des impacts sociaux des mégaprojets industriels, réalisé à l'Université de Sydney en Australie. christiane.gagnon@uqac.ca

\section{MAJELLA-J GAUTHIER}

Majella-J Gauthier est géographe et professeur émérite en sciences humaines à l'Université du Québec à Chicoutimi. majella.gauthier@uqac.ca 